Check for updates

Cite this: Chem. Commun., 2017, 53,11360

Received 1st September 2017

Accepted 26th September 2017

DOI: $10.1039 / c 7 c c 06854 a$

rsc.li/chemcomm

\section{Disentangling the effect of seed size and crystal habit on gold nanoparticle seeded growth $\dagger$}

\author{
Guillermo González-Rubio, ${ }^{\text {abc }}$ Thais Milagres de Oliveira, ${ }^{d}$ Thomas Altantzis, ${ }^{d}$ \\ Andrea La Porta, ' Andrés Guerrero-Martínez, b Sara Bals, d Leonardo Scarabelli (iD *aef \\ and Luis. M. Liz-Marzán (iD *acg
}

\begin{abstract}
Oxidative etching was used to produce gold seeds of different sizes and crystal habits. Following detailed characterization, the seeds were grown under different conditions. Our results bring new insights toward understanding the effect of size and crystallinity on the growth of anisotropic particles, whilst identifying guidelines for the optimisation of new synthetic protocols of predesigned seeds.
\end{abstract}

Seed-mediated growth of metal nanoparticles was introduced by the turn of the century, along with the first colloidal synthesis of gold nanorods. ${ }^{1}$ Almost two decades later, we are now able to prepare a wide library of nanoparticles using this versatile method, ranging from isotropic platonic solids through anisotropic wires or star-like particles. ${ }^{2,3}$ While growth conditions have been meticulously optimised for maximum shape-yield and size monodispersity, seed preparation remains pretty much identical to the original recipe. It is however wellknown that a close relationship exists between the shape, size and crystallinity of the seeds and those of the resulting product, rendering this consideration even more significant. ${ }^{4-6}$ In particular, it was proposed that the crystallinity of the seeds (i.e. the presence of twin defects) is maintained during growth, and seed size was thought to radically influence the reaction kinetics and thermodynamics, as well as the final morphology of the product. ${ }^{7}$ However, the shortage of currently available instruments and methodologies to adequately characterise

\footnotetext{
${ }^{a}$ Bionanoplasmonics Laboratory, CIC biomaGUNE, Paseo de Miramón 182, 20014 Donostia-San Sebastián, Spain. E-mail: llizmarzan@cicbiomagune.es

${ }^{b}$ Departamento de Quimica Física I, Universidad Complutense de Madrid, Avda. Complutense s/n, 28040, Madrid, Spain

${ }^{c}$ Ciber de Bioingeniería, Biomateriales y Nanomedicina, Ciber-BBN, 20014 Donostia-San Sebastián, Spain

${ }^{d}$ EMAT-University of Antwerp, Groenenborgerlaan 171, B-2020 Antwerp, Belgium

${ }^{e}$ Department of Chemistry and Biochemistry, UCLA, Los Angeles, California 90095, USA.E-mail: lscarabelli@ucla.edu

${ }^{f}$ California NanoSystems Institute, UCLA, Los Angeles, California 90095, USA

${ }^{g}$ Ikerbasque, Basque Foundation for Science, 48013 Bilbao, Spain

$\dagger$ Electronic supplementary information (ESI) available: Experimental details and additional spectra and electron microscopy images. See DOI: 10.1039/c7cc06854a
}

such small structures, hinders further detailed investigation. We recently reported the synthesis of gold nanotriangles, in which the amount of twin defects present in the seeds was identified as the key parameter affecting the resulting shapeyield. ${ }^{8}$ In this context, aging of the seed solution appears to be crucial toward a successful synthesis (ESI, $\dagger$ Fig. S1). In a more recent report, we demonstrated that a mild thermal treatment of the seeds resulted in a gradual increase of shape-yield for penta-twinned particles (rods, bipyramids, decahedra), from roughly $30 \%$ to over $90 \% .{ }^{9}$ This and other recent experimental evidences confirm that a better understanding of seed transformation and more reliable methods to control their fabrication will represent a significant step forward in the synthesis of gold nanoparticles. ${ }^{10-12}$ We present here a new methodology that allows us to carefully analyse the role of twin defects in the seeds, on the growth of gold nanoparticles. In particular, we examined the evolution of single-crystal and mono-twinned seeds when grown under conditions typically used for nanooctahedra and nanotriangles, i.e. single-crystal and mono-twinned products. We aim at understanding whether the presence of structural defects can hinder the formation of certain shapes, at which stage of the growth process defects can be introduced, and the role of seed size in the outcome of the synthesis.

The main idea behind this work is that a better control over the crystallographic structure of the starting seeds can be obtained by means of a top-down oxidative etching approach (Fig. 1A). We thus started from the synthesis of high-quality gold (single-crystal) nanorods and (mono-twinned) nanotriangles, following optimised literature protocols. ${ }^{8,13,14}$ The particles were etched using $\mathrm{Au}^{\text {III }}$ as oxidant, in a mixture of cetyltrimethylammonium chloride (CTAC) and sodium bromide, so that particles were gradually reduced to the desired size. ${ }^{12,15}$ The choice of nanorods (length: $59 \pm 5 \mathrm{~nm}$, diameter: $25 \pm 2 \mathrm{~nm}$, yield: $95 \%$ ) and nanotriangles (edge length: $59 \pm 3 \mathrm{~nm}$, yield: $93 \%$ ) as starting materials was motivated from the need to prepare single-crystal and monotwinned seeds, respectively. To address the effect of size we prepared $20 \mathrm{~nm}$ and $12 \mathrm{~nm}$ seeds, both from nanorods (20NR and $12 \mathrm{NR}$ ) and from nanotriangles (20NT and $12 \mathrm{NT}$ ). The four 


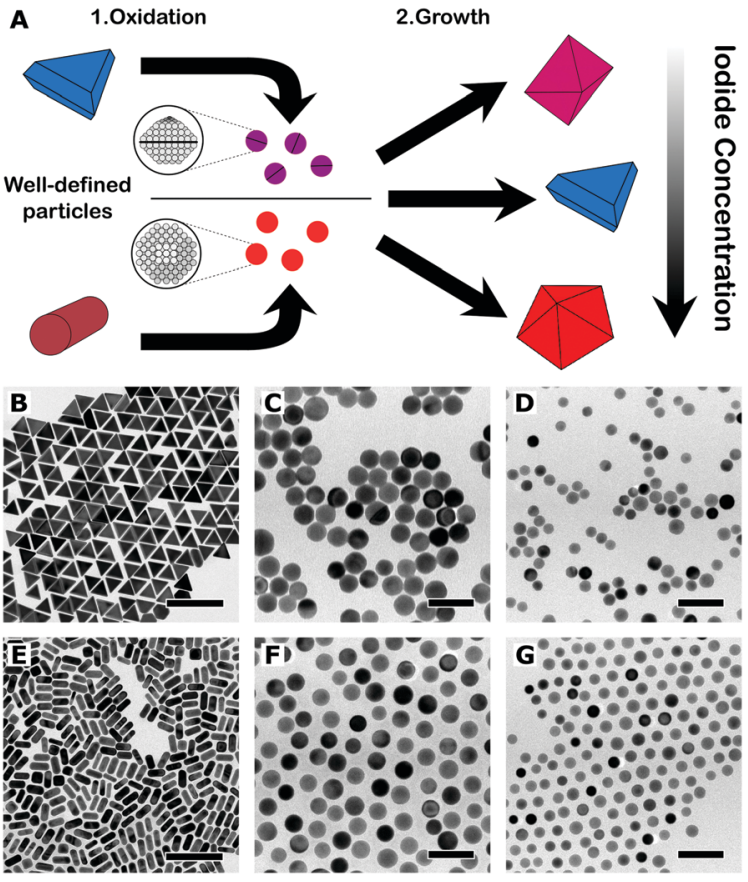

Fig. 1 (A) Schematic representation of the experimental procedure (see text). (B-G) TEM images of the starting nanotriangles (B) and nanorods (E), and the seeds prepared by sequential oxidation: 20NT (C), 12NT (D), 20NR (F) and 12NR (G). Scale bars: B and E: $200 \mathrm{~nm} ; \mathrm{C}, \mathrm{D}, \mathrm{F}$ and G: $50 \mathrm{~nm}$.

resulting seed samples were characterised by transmission electron microscopy (TEM), as summarised in Table 1.

Representative TEM images of the starting nanotriangles and nanorods are shown in Fig. 1B and E, demonstrating a low dispersion in both size and shape, in agreement with the narrow plasmon bands registered in the corresponding UV-vis spectra (ESI, $\dagger$ Fig. S2). It should be noted that standard characterisation tools do not allow us to fully address the crystal habit of the different seeds (Fig. 1C, D, F and G). Instead, we used annular dark-field scanning transmission electron microscopy (ADF-STEM) at relatively low magnification. By inspection of the residual diffraction contrast in hundreds of different NPs, we were able to quantify the amount of twinned particles in each sample (details of the procedure can be found in the ESI, $\dagger$ Fig. S3). ${ }^{9}$ Interestingly, extensive analysis of the degree of twinning in the seeds resulting from nanotriangles oxidation indicated that the percentage of mono-twinned particles was reduced by half when the average size was decreased from $20 \mathrm{~nm}$ to $12 \mathrm{~nm}$, from $51 \%$ to $29 \%$ (see Table 1). This suggests that the oxidative process may be used to remove twin-defects from

Table 1 Crystal structure characterisation of the four seeds, comparing results obtained for the seeds before (ADF-STEM) and after (TEM) silver coating. For each sample, at least 300 nanoparticles were measured

\begin{tabular}{llll}
\hline & $\begin{array}{l}\text { Size } \\
(\mathrm{nm})\end{array}$ & $\begin{array}{l}\text { Single-crystal: mono- } \\
\text { twinned (ADF-STEM), \% }\end{array}$ & $\begin{array}{l}\text { Single-crystal : mono- } \\
\text { twinned (Ag coating), \% }\end{array}$ \\
\hline 12NT & $12 \pm 2$ & $71: 29$ & $71: 29$ \\
12NR & $12 \pm 1$ & $99: 1$ & $99: 1$ \\
20NT & $20 \pm 1$ & $49: 51$ & $48: 52$ \\
20NR & $19 \pm 1$ & $99: 1$ & $99: 1$
\end{tabular}

preformed seeds. In order to confirm this hypothesis, we carried out a simple control experiment by epitaxially growing silver shells on both 12NT and 20NT seed solutions (Fig. 2), so that the crystallinity of the seeds is maintained, and the morphology of the resulting core-shell nanoparticles would be an indication of the original seed crystal habit. ${ }^{16}$ UV-vis spectral analysis (Fig. 2C) of the obtained colloids shows two main plasmon bands, one centred at $450 \mathrm{~nm}$ and the second one around $515 \mathrm{~nm}$ (higher energy modes correspond to multipoles and are not discussed further). Whereas the low energy band dominates in the sample grown from 20NT, the band at $450 \mathrm{~nm}$ is more intense for the particles grown from 12NT. In accordance with previous reports, as well as with the corresponding TEM analysis (Fig. 2D-E), the $515 \mathrm{~nm}$ band can be assigned to nanobipyramids with triangular base, whereas the band at $450 \mathrm{~nm}$ corresponds to nanocubes. ${ }^{17}$ By using electron tomography in high angle annular dark field STEM (HAADFSTEM) mode, we were able to determine the three-dimensional shape of the particles (ESI, $\uparrow$ Fig. S4) and confirm the presence of a single twin plane in the bipyramids (Fig. 2F). A similar control experiment was performed for 20NR and 12NR seeds (results shown in ESI, $†$ Fig. S5); as expected, the resulting Au@Ag coreshell particles were nanocubes in both cases, with almost identical UV-vis spectra. The percentages of single-crystal and mono-twinned particles estimated from both methods (ADFSTEM and silver coating) are in excellent agreement (Table 1). These data confirm that silver coating represents a simple indirect
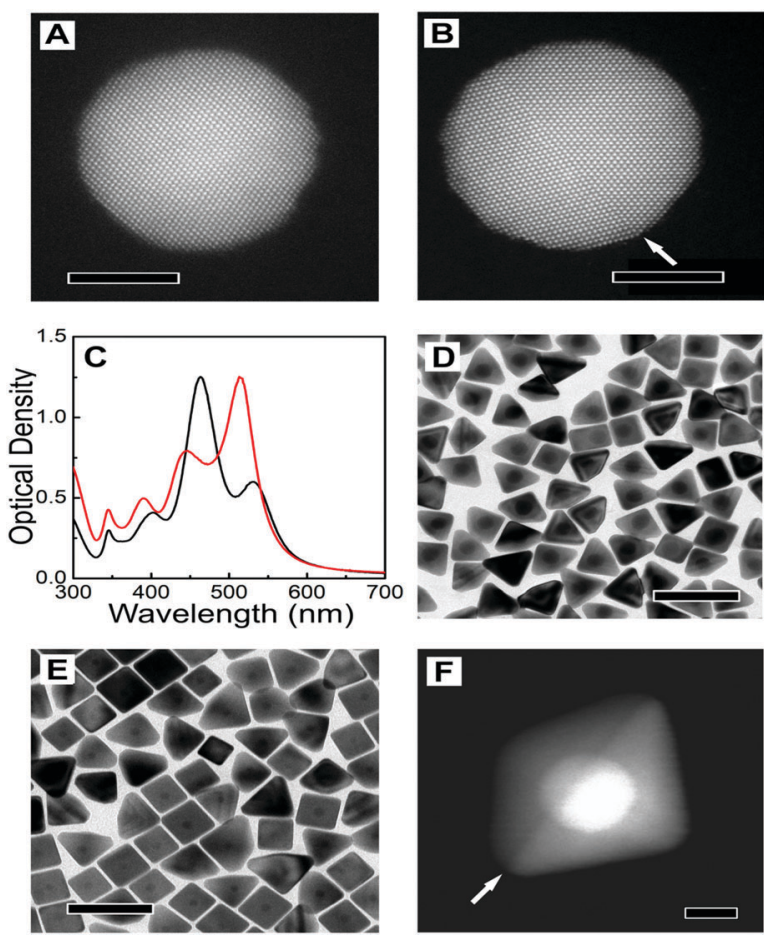

Fig. 2 ( $A$ and $B$ ) High-resolution TEM images of single-crystal (A, 12NR) and mono-twinned (B, 12NT) seeds. (C) UV-vis spectra of 20NT (red line) and 12NT (black line) seeds coated with silver. ( $D$ and $E$ ) Representative TEM images of 20NT (D) and 12NT (E) seeds after silver growth. (F) HAADFSTEM image of an Au seed coated with Ag, forming a triangular-base nanobipyramid. The white arrows in (B) and (F) point to the corresponding twin planes. Scale bars: (A and B) $5 \mathrm{~nm}$; (D and E) $100 \mathrm{~nm}$; (F) $10 \mathrm{~nm}$. 
method that can be safely used to estimate the crystal habit of seed solutions when more advanced and costly approaches are not available.

Upon preparation and characterisation of the four seed samples, we investigated seeded growth with $\mathrm{Au}$, under different experimental conditions, which we selected taking the following into account: (i) synthetic protocols for differently shaped products, with different crystal habits easy to identify by standard TEM analysis and; (ii) the protocols should share most of the chemical compounds in the growth solution (i.e., the same surfactant, reducing agent and temperature); (iii) the final particles should be all thermodynamic (as opposed to kinetic) products; ${ }^{18}$ (iv) Au should be the only metal involved (no $\mathrm{Ag}^{+}$ addition). Following these criteria, we carried out seeded growth on the four different seed samples, using CTAC as surfactant, ascorbic acid as reducing agent and iodide as shape-inducing agent (ESI, $\dagger$ Experimental section). The reaction conditions were optimised in such a way that a small change in iodide concentration would induce the growth of nanooctahedra $\left(\left[\mathrm{I}^{-}\right] \approx 50 \mu \mathrm{M}\right)$ or nanotriangles ([ $\left.\left.\mathrm{I}^{-}\right] \approx 75 \mu \mathrm{M}\right)$ : in fact, depending on the concentration, iodide has been demonstrated to either be a shape directing agent and/or induce the formation of twin defects during particle growth. ${ }^{8,19,20}$ Growth of the seeds in the absence of iodide was found to result in undefined shapes, which would complicate the interpretation of the data (ESI, $\dagger$ Fig. S6). We show in Fig. 3 the results from seeded growth on both $20 \mathrm{~nm}$ seed samples. We find that growth on 20NT leads to formation of nanotriangles, even at low iodide content (Fig. 3C), while a higher concentration does not significantly influence the final product morphology (Fig. 3E and G). Moreover, we did not observe the formation of penta-twinned by-products, unless the concentration of iodide was increased up to $500 \mu \mathrm{M}$ (ESI, $\dagger$ Fig. S7). In the case of $20 \mathrm{NR}$, nanooctahedra were obtained as $99 \%$ of the final product using $50 \mu \mathrm{M}$ of iodide, as expected for the growth on single-crystal seeds (Fig. 3D). However, the content in nanotriangles remained below $45 \%$, even when adding $100 \mu \mathrm{M}$ iodide to the growth solution (Fig. $3 \mathrm{H}$ ), and a transition of the majority of the product into mono-twinned particles required a higher iodide concentration $(500 \mu \mathrm{M}$, see ESI, $\dagger$ Fig. S7). To further support our analysis, we estimated the percentage of single-crystal, mono-twinned and penta-twinned particles in each product, by identifying the number of nanotriangles, nanooctahedra and nanodecahedra in TEM images (ESI, $\dagger$ Fig. S8). The results reported in Table 2 indicate that the amount of single-crystal particles resulting from the growth of 20NR and 20NT is reduced as iodide concentration increases (Table 1 and Fig. 2). Overall, these data suggest that $20 \mathrm{~nm}$ seeds tend to retain their crystallographic structure during growth, unless a strong driving force towards defect formation (e.g. high iodide concentration) is applied. A parallel study was carried out on $12 \mathrm{~nm}$ seeds (ESI, $\uparrow$ Fig. S9-S11). Interestingly, even though $12 \mathrm{NT}$ contains $73 \%$ single-crystal seeds, only $13 \%$ nanooctahedra were grown at low iodide concentration ( $c f$. Tables 1 and 2). This suggests that defects can be readily created, even at low iodide concentration. Similarly, nanotriangles were obtained as a majority population upon seeded growth on 12NR seeds using only $50 \mu \mathrm{M}$ iodide (Fig. S9F, ESI $\dagger$ ). Another
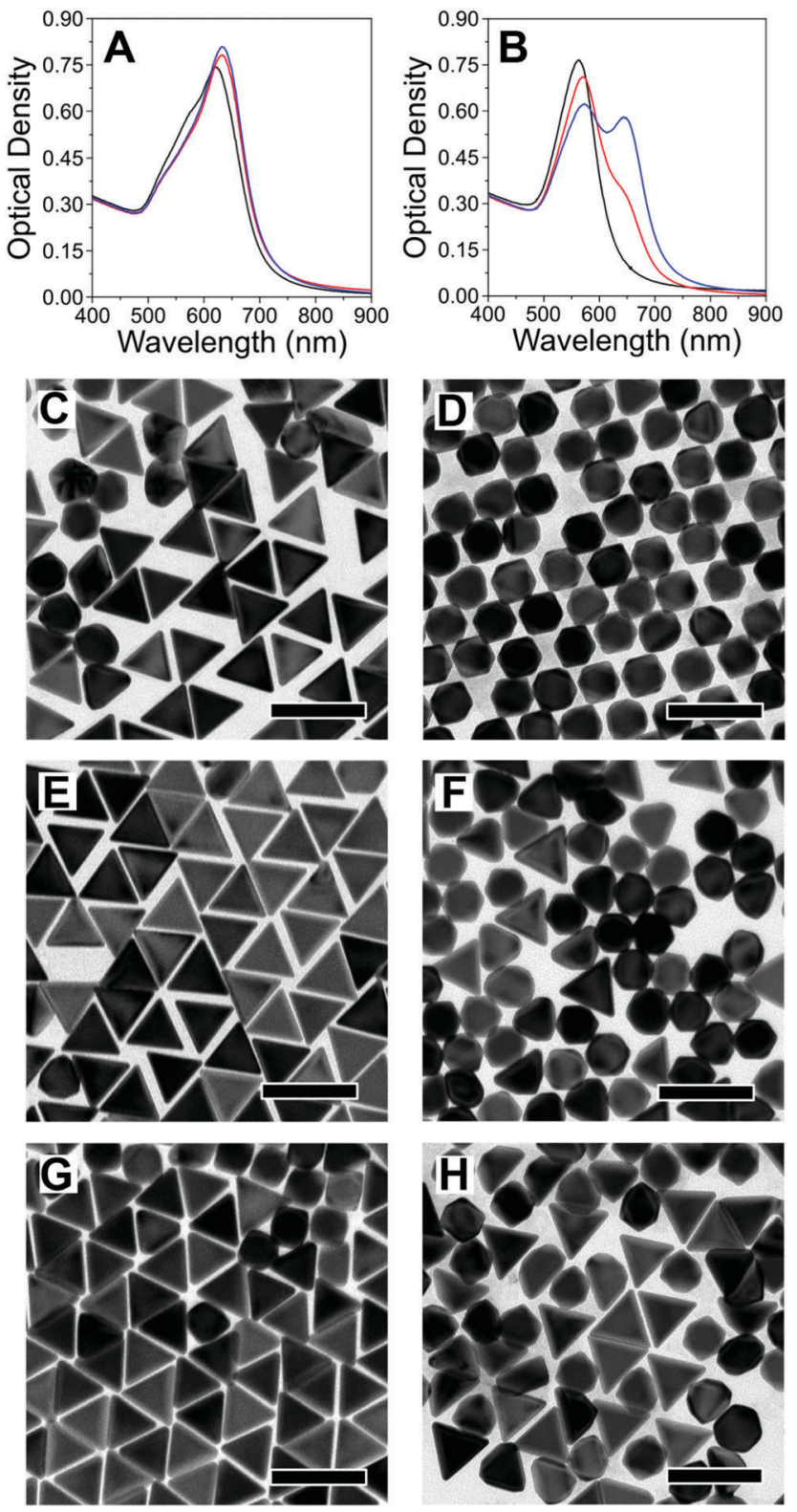

Fig. 3 (A and B) UV-vis spectra of 20NT (A) and 20NR (B), grown in the presence of different concentrations of iodide: $50 \mu \mathrm{M}$ (black), $75 \mu \mathrm{M}$ (red) and $100 \mu \mathrm{M}$ (blue). (C-H) TEM analysis of 20NT (left) and 20NR (right) grown with different iodide concentrations: $50 \mu \mathrm{M}$ ( $C$ and $D), 75 \mu \mathrm{M}$ ( $E$ and $\mathrm{F}$ ), $100 \mu \mathrm{M}(\mathrm{G}$ and $\mathrm{H})$. Scale bars: $100 \mathrm{~nm}$. (Images at different magnifications are shown in the ESI, † Fig. S12 and S13).

Table 2 Crystal habit of seeded-grown particles. For each sample, we counted at least 250 nanoparticles. The total differs from 100\% because the shape could not be identified for a small fraction of particles

\begin{tabular}{llll}
\hline & \multicolumn{3}{l}{ Nanooctahedra : nanotriangles : nanodecahedra } \\
\cline { 2 - 4 } & {$\left[\mathrm{I}^{-}\right]=50 \mu \mathrm{M}$} & {$\left[\mathrm{I}^{-}\right]=75 \mu \mathrm{M}$} & {$\left[\mathrm{I}^{-}\right]=100 \mu \mathrm{M}$} \\
\hline 12NT & $13: 66: 10$ & $7: 63: 15$ & $4: 54: 29$ \\
$12 \mathrm{NR}$ & $27: 41: 0$ & $3: 59: 5$ & $3: 49: 9$ \\
$20 \mathrm{NT}$ & $26: 64: 5$ & $20: 64: 9$ & $11: 70: 5$ \\
$20 \mathrm{NR}$ & $99: 1: 0$ & $59: 30: 0$ & $42: 44: 0$
\end{tabular}


important observation concerns the formation of penta-twinned particles for both $12 \mathrm{NR}$ and $12 \mathrm{NT}$ seeds, at relatively low iodide concentrations as compared to the corresponding $20 \mathrm{~nm}$ seeds (Table 2 and Fig. S9, ESI $\dagger$ ). Overall, we find that the crystal habit of smaller seeds can evolve during the growth process, resulting in the introduction of both mono-twinned and penta-twinned structures.

The use of a simple chemical etching method on welldefined single-crystal and mono-twinned nanoparticles allowed us to obtain seeds with selected size and crystallinity, which were quantitatively characterised using both direct electron microscopy (ADF-STEM) analysis and indirectly by silver coating into distinct morphologies. Seeded growth under specific conditions was then used to separately investigate the effect of size and twinning on the shape evolution of gold nanoparticles: we clearly found that twin defects can be introduced (but not removed) during seeded growth, and that it is significantly easier to introduce defects in $12 \mathrm{~nm}$ seeds than in larger $20 \mathrm{~nm}$ seeds. Overall, the presented data suggest that optimization of protocols for better defined seeds toward formation of gold nanotriangles or nanooctahedra, should focus on nanoparticles with larger diameter, so as to achieve a better control over crystallographic structure and its evolution during growth.

As a first example of how the development of better seeds will lead toward the optimisation of more reliable and reproducible synthetic protocols, we re-optimised the synthesis of gold nanotriangles by using $20 \mathrm{~nm}$ seeds prepared with a bottom-up approach (see ESI, $\dagger$ Experimental section). ${ }^{21,22}$ The hypothesis is that using bigger seeds we would be able to achieve a better control over the formation of defects during growth, thereby maximizing the population of mono-twinned particles, while avoiding multi-twinned structures. Indeed, we obtained gold nanotriangles with a shape yield of $70 \%$ (ESI, $\dagger$ Fig. S16), a significant increase compared to the percentage usually achieved with the standard protocol (50-55\%). ${ }^{8}$

This work was funded by the Spanish MINECO (grant \# MAT2013-46101-R, Ramon y Cajal fellowship to A. G.-M. and FPI fellowship to G. G.-R.). Financial support is acknowledged from the European Commission (EUSMI, 731019). S. B. acknowledges financial support from the European Research Council (ERC Starting Grant \# 335078-COLOURATOMS). T. A. acknowledges a postdoctoral grant from Research Foundation Flanders (FWO, Belgium).

\section{Conflicts of interest}

There are no conflicts to declare.

\section{Notes and references}

1 N. R. Jana, L. Gearheart and C. J. Murphy, J. Phys. Chem. B, 2001, 105, 4065-4067.

2 S. E. Lohse, N. D. Burrows, L. Scarabelli, L. M. Liz-Marzán and C. J. Murphy, Chem. Mater., 2014, 26, 34-43.

3 L. Scarabelli, PhD dissertation, Universidade de Vigo, 2016.

4 M. Liu and P. Guyot-Sionnest, J. Phys. Chem. B, 2005, 109, 22192-22200.

5 M. Grzelczak, J. Pérez-Juste, P. Mulvaney and L. M. Liz-Marzán, Chem. Soc. Rev., 2008, 37, 1783-1791.

6 A. Gole and C. J. Murphy, Chem. Mater., 2004, 16, 3633-3640.

7 L. M. Liz-Marzán and M. Grzelczak, Science, 2017, 356, 1120-1121.

8 L. Scarabelli, M. Coronado-Puchau, J. J. Giner-Casares, J. Langer and L. M. Liz-Marzán, ACS Nano, 2014, 8, 5833-5842.

9 A. Sánchez-Iglesias, N. Winckelmans, T. Altantzis, S. Bals, M. Grzelczak and L. M. Liz-Marzán, J. Am. Chem. Soc., 2017, 139, 107-110.

10 A. Ruditskiy, M. Vara, H. Huang and Y. Xia, Chem. Mater., 2017, 29, 5394-5400.

11 M. Lin, G.-H. Kim, J.-H. Kim, J.-W. Oh and J.-M. Nam, J. Am. Chem. Soc., 2017, 139, 10180-10183.

12 M. N. O'Brien, M. R. Jones, K. A. Brown and C. A. Mirkin, J. Am. Chem. Soc., 2014, 136, 7603-7606.

13 L. Scarabelli, A. Sánchez-Iglesias, J. Pérez-Juste and L. M. LizMarzán, J. Phys. Chem. Lett., 2015, 6, 4270-4279.

14 L. Scarabelli, M. Grzelczak and L. M. Liz-Marzán, Chem. Mater., 2013, 25, 4232-4238.

15 E. Carbó-Argibay, B. Rodríguez-González, J. Pacifico, I. PastorizaSantos, J. Pérez-Juste and L. M. Liz-Marzán, Angew. Chem., Int. Ed., 2007, 46, 8983-8987.

16 S. Gómez-Graña, B. Goris, T. Altantzis, C. Fernández-López, E. CarbóArgibay, A. Guerrero-Martínez, N. Almora-Barrios, N. López, I. PastorizaSantos, J. Pérez-Juste, S. Bals, G. Van Tendeloo and L. M. Liz-Marzán, J. Phys. Chem. Lett., 2013, 4, 2209-2216.

17 B. J. Wiley, Y. Xiong, Z.-Y. Li, Y. Yin and Y. Xia, Nano Lett., 2006, 6, $765-768$.

18 Y. Xia, X. Xia and H.-C. Peng, J. Am. Chem. Soc., 2015, 137, 7947-7966.

19 M. R. Langille, M. L. Personick, J. Zhang and C. A. Mirkin, J. Am. Chem. Soc., 2012, 134, 14542-14554.

20 P.-J. Chung, L.-M. Lyu and M. H. Huang, Chem. - Eur. J., 2011, 17, 9746-9752.

21 Y. Zheng, X. Zhong, Z. Li and Y. Xia, Part. Part. Syst. Charact., 2014, 31, 266-273.

22 C. Hanske, G. González-Rubio, C. Hamon, P. Formentín, E. Modin, A. Chuvilin, A. Guerrero-Martínez, L. F. Marsal and L. M. LizMarzán, J. Phys. Chem. C, 2017, 121, 10899-10906. 